\title{
A scientific note on improved isolation methods for Melissococcus plutonius from diseased Apis mellifera larvae
}

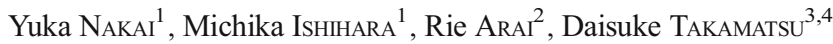 \\ ${ }^{1}$ Saitama Prefectural Chuo Livestock Hygiene Service Center, 107-1 Besshocho, Kita-ku, Saitama, Saitama 331-0821, Japan \\ ${ }^{2}$ Livestock Safety Division, Department of Agriculture and Forestry, Saitama Prefectural Government, 3-15-1 Takasago, Urawa-ku, \\ Saitama, Saitama 330-9301, Japan \\ ${ }^{3}$ Division of Bacterial and Parasitic Disease, National Institute of Animal Health, National Agriculture and Food Research \\ Organization, 3-1-5 Kannondai, Tsukuba, Ibaraki 305-0856, Japan \\ ${ }^{4}$ The United Graduate School of Veterinary Sciences, Gifu University, 1-1 Yanagido, Gifu, Gifu 501-1193, Japan
}

Received 14 November 2017 - Revised 6 February 2018 - Accepted 7 March 2018

European foulbrood / Melissococcus plutonius / Apis mellifera / Enterococcus faecalis / royal jelly

European foulbrood (EFB) caused by Melissococcus plutonius is a significant infectious disease of honey bee brood. EFB is typically diagnosed based on the visual inspection of brood combs and detection of diseased larvae; however, since symptoms of EFB may easily be confused with other diseases or abnormalities in larvae, verification of the presence of M. plutonius in diseased larvae is required for a reliable diagnosis of EFB in many cases. The isolation and storage of M. plutonius strains are also important for future EFB studies. However, difficulties are sometimes associated with the isolation of $M$. plutonius from diseased larvae by culture methods due to the growth of secondary bacteria associated with EFB.

In an inspection of foulbrood on May 16, 2017, an abnormal Apis mellifera colony was detected in an apiary in Saitama prefecture, Japan. The colony showed irregular capping, and dead larvae were observed in uncapped cells. Gram-positive cocci were detected in dead larvae by microscopy. Furthermore, an M. plutonius -specific DNA fragment was found in dead larvae by PCR (Arai et al. 2014); however, due to the vigorous growth of

Electronic supplementary material The online version of this article (https://doi.org/10.1007/s13592-018-0572-4) contains supplementary material, which is available to authorized users.

Corresponding author: D. Takamatsu, p1013dt@affrc.go.jp

Manuscript editor: Peter Rosenkranz
Enterococcus faecalis, a common secondary invader of $\mathrm{EFB}$, on agar medium in anaerobic conditions, it was not possible to isolate M. plutonius from larvae using culture methods in the first examination in the laboratory. To prevent the growth of some secondary bacteria from larvae samples, the addition of nalidixic acid to agar medium at a final concentration of $3 \mu \mathrm{g} / \mathrm{ml}$ has been suggested (Forsgren et al. 2013); however, since nalidixic acid does not work on E. faecalis (Hornitzky and Smith 1998), it was not suitable for the selective isolation of M. plutonius in this case. Thus, in order to eliminate secondary bacteria from the samples, we spread out homogenates of dead larvae on glass slides, allowed the smears to dry, and stored them for 1 month in the laboratory (temperature, approximately $20-35^{\circ} \mathrm{C}$ ) by referring to the Manual of Diagnostic Tests and Vaccines for Terrestrial Animals (http://www.oie.int/fileadmin/ Home/eng/Health_standards/tahm/2.02.03_EUROPEAN_FOULBROOD.pdf). Viable E. faecalis cells decreased during storage, and M. plutonius was isolated from the smears (Online Resource 1). These results confirmed the usefulness of this method for the isolation of M. plutonius from larvae contaminated with secondary bacteria; however, since it was a time-consuming method, we herein conducted two experiments using the same larvae samples and devised time-saving and timeefficient methods to isolate M. plutonius from enterococci-contaminated samples.

In the first experiment to investigate the minimum storage time required to eliminate E. faecalis, we prepared dried smears of dead larvae as described above 
and stored them for $1,3,7,10,14$, and 21 days in the laboratory (temperature, approximately $25-35^{\circ} \mathrm{C}$ ). After storage, each smear was mixed with $50 \mu \mathrm{l}$ of sterilized saline on glass slides, and bacteria that survived in the smears were isolated by streaking $10 \mu \mathrm{l}$ of the saline and smear mixture on KSBHI agar plates (Arai et al. 2012) and incubating the plates at $37^{\circ} \mathrm{C}$ under anaerobic conditions. Isolated bacteria were identified using M. plutonius -specific PCR (Arai et al. 2014). As summarized in Table I, Enterococcus -like bacteria from the smear stored for only 1 day vigorously grew on KSBHI agar, and M. plutonius -like colonies were not observed. However, viable Enterococcus -like bacteria markedly decreased in samples stored for 3 or more days, and M. plutonius was predominantly or purely isolated from the samples (Table I and Online Resource 2), suggesting that storage for 3 days is sufficient to reduce Enterococcus from dried smears of larvae samples.

Takamatsu et al. (2017) recently showed that M. plutonius strains were more resistant to the antimicrobial activity of royal jelly (RJ) than other bacterial strains including an E. faecalis strain. In the previous study, most of the $M$. plutonius strains tested were not killed completely within $24 \mathrm{~h}$, even in 50\% RJcontaining broth, whereas viable E. faecalis cells became undetectable in $6.25-12.5 \%$ RJ-containing broth after a $24-\mathrm{h}$ incubation at $35{ }^{\circ} \mathrm{C}$. These findings prompted us to employ the antimicrobial activity of RJ to eliminate secondary invaders, and in the second experiment, larval homogenates were incubated in $0,6.25,12.5,25,50$, and $75 \%$ RJ-containing KSBHI broth at $37{ }^{\circ} \mathrm{C}$ for $24 \mathrm{~h}$. After the incubation, bacteria that survived in the RJ-containing broth were isolated by spreading $200 \mu \mathrm{l}$ of the broth on KSBHI agar plates and incubating the plates at $37{ }^{\circ} \mathrm{C}$ under anaerobic conditions.

Enterococcus -like bacteria vigorously grew from samples incubated in broth without RJ $(0 \%)$ and that containing 6.25 and $12.5 \% \mathrm{RJ}$, and M. plutonius was not isolated from the samples (Table I). Conversely, M. plutonius was predominantly or purely isolated from samples incubated in 25,50 , and $75 \%$ RJ-containing broth (Table I

Table I. Isolation of M. plutonius from secondary invader-contaminated larvae

Pretreatment method for samples ${ }^{\mathrm{a}}$

Isolation $^{\mathrm{b}}$

M. plutonius

Enterococcus -like bacteria

Storage of dried larval smears for at $37^{\circ} \mathrm{C}$ in $\mathrm{KSBHI}$ broth

24-h incubation of larval samples

24-h incubation of larval samples
at $37^{\circ} \mathrm{C}$ in KSBHI broth

(n)

1 day
3 days
7 days
10 days
14 days
21 days
without RJ
with $6.25 \% \mathrm{RJ}$
with $12.5 \% \mathrm{RJ}$
with $25 \% \mathrm{RJ}$
with $50 \% \mathrm{RJ}$
with $75 \% \mathrm{RJ}$

Inoculation of larval homogenates on KSBHI agar with $3 \mu \mathrm{g} / \mathrm{ml} \mathrm{NA}$

$\begin{array}{ll}- & + \\ +^{\mathrm{s}} & +^{\mathrm{s}} \\ + & - \\ + & +^{\mathrm{s}} \\ + & +^{\mathrm{s}} \\ + & - \\ - & + \\ - & + \\ - & + \\ + & +^{\mathrm{s}} \\ + & - \\ + & - \\ - & +\end{array}$

${ }^{\text {a }} R J$, royal jelly; $N A$, nalidixic acid. Fresh RJ used in this study was purchased from Yamada Bee Farm (Tomata, Okayama, Japan) and stored in a freezer at $\leq-80^{\circ} \mathrm{C}$ until used. The absence of $M$. plutonius in RJ used in this study was confirmed by culturing RJ on $\mathrm{KSBHI}$ agar under anaerobic conditions. Each pretreated sample was inoculated onto a single KSBHI agar plate for the isolation of bacteria

${ }^{\mathrm{b}}+$, Innumerable colonies of the corresponding bacteria appeared on KSBHI agar; ${ }^{\mathrm{s}}$, Less than 200 colonies appeared on KSBHI agar; -, No colonies of the corresponding bacteria were observed on KSBHI agar (Online Resources 2 and 3)

${ }^{\mathrm{c}}$ M. plutonius -like colonies grown on KSBHI agar were identified as M. plutonius by species-specific PCR (Arai et al. 2014) 
and Online Resource 3). These results demonstrated for the first time that the incubation of larval homogenates in high-concentration RJ-containing broth is effective for the selective isolation of M. plutonius from enterococci-contaminated larvae.

In the present study, we found that a 3-day storage at room temperature was sufficient to reduce enterococci from dried larval smears and that the antimicrobial activity of RJ was useful for the selection of $M$. plutonius from larvae containing enterococci. RJ only needed $24 \mathrm{~h}$ to select M. plutonius. In this case, the growth of Enterococcus -like bacteria was not inhibited on $3 \mu \mathrm{g} / \mathrm{ml}$ nalidixic acid-containing KSBHI agar (Table I) which confirms the report of Hornitzky and Smith (1998). However, since this antibiotic is expected to be effective against other secondary bacteria like Paenibacillus alvei, the incubation of larval homogenates in RJ-containing broth and subsequent cultivation of samples on nalidixic acidcontaining agar media may be more effective for the isolation of M. plutonius from EFB cases.

It is important to note that the M. plutonius strains analyzed to date have been grouped into three clonal complexes (CC3, $\mathrm{CC} 12$, and $\mathrm{CC} 13)$ by multilocus sequence typing (Haynes et al. 2013; Budge et al. 2014; Takamatsu et al. 2014). Among these groups, CC3 strains exhibited the strongest resistance to the antimicrobial activity of RJ (Takamatsu et al. 2017). Since the causative M. plutonius strain in this EFB case was sequence type 3 of $\mathrm{CC} 3$, the strain may be efficiently selected by RJ in this study. In addition, the degree of antimicrobial activity of RJ may be different according to the origins (Takamatsu et al. 2017). Therefore, further studies using larvae samples of other EFB cases and RJ of different origins are needed in order to verify the usefulness of the selective isolation method for M. plutonius proposed in this study.

\section{AUTHORS' CONTRIBUTIONS}

YN and MI: Diagnosed the EFB case described in this study and performed experiments; RA: Designed the first experiment; DT: Designed the second experiment and contributed to writing the manuscript. All authors read and approved the final manuscript.

\section{COMPLIANCE WITH ETHICAL STANDARDS}

Conflict of interest The authors declare that they have no conflict of interest.

Une note scientifique sur l'amélioration des méthodes d'isolement pour Melissococcus plutonius de larves malades d 'Apis mellifera

Eine wissenschaftliche Notiz über eine verbesserte Methode zur Isolierung von Melissococcus plutonius aus kranken Apis mellifera Larven

\section{REFERENCES}

Arai, R., Tominaga, K., Wu, M., Okura, M., Ito, K., Okamura, N., Onishi, H., Osaki, M., Sugimura, Y., Yoshiyama, M., Takamatsu, D. (2012) Diversity of Melissococcus plutonius from honeybee larvae in Japan and experimental reproduction of European foulbrood with cultured atypical isolates. PLoS One 7 (3), e33708

Arai, R., Miyoshi-Akiyama, T., Okumura, K., Morinaga, Y., Wu, M., Sugimura, Y., Yoshiyama, M., Okura, M., Kirikae, T., Takamatsu, D. (2014) Development of duplex PCR assay for detection and differentiation of typical and atypical Melissococcus plutonius strains. J. Vet. Med. Sci. 76 (4), 491-498

Budge, G. E., Shirley, M. D. F., Jones, B., Quill, E., Tomkies, V., Feil, E. J., Brown, M. A., Haynes, E. G. (2014) Molecular epidemiology and population structure of the honey bee brood pathogen Melissococcus plutonius . ISME J. 8 (8), 1588-1597

Forsgren, E., Budge, G. E., Charrière J.-D., Hornitzky, M. A. Z. (2013) Standard methods for European foulbrood research. J. Apic. Res. 52 (1), 1-14

Haynes, E., Helgason, T., Young, J. P. W., Thwaites, R., Budge, G. E. (2013) A typing scheme for the honeybee pathogen Melissococcus plutonius allows detection of disease transmission events and a study of the distribution of variants. Environ. Microbiol. Rep. 5 (4), 525-529

Hornitzky, M. A. Z., Smith, L. (1998) Procedures for the culture of Melissococcus pluton from diseased brood and bulk honey samples. J. Apic. Res. 37 (4): 293-294

Takamatsu, D., Morinishi, K., Arai, R., Sakamoto, A., Okura, M., Osaki, M. (2014) Typing of Melissococcus plutonius isolated from European and Japanese honeybees suggests spread of sequence types across borders and between different Apis species. Vet. Microbiol. 171 (1-2), 221-226

Takamatsu, D., Osawa, A., Nakamura, K., Yoshiyama, M., Okura, M. (2017) High-level resistance of Melissococcus plutonius clonal complex 3 strains to antimicrobial activity of royal jelly. Environ. Microbiol. Rep. 9 (5), 562-570 